capacity - it's cheap and can be mass-produced," says Emanuel Petricoin, co-director with Lance Liotta of the Center for Applied Proteomics and Molecular Medicine at George Mason University in Manassas, Virginia. "The problem is that it has high background fluorescence and you have to come up with different labelling strategies to get around that." One solution uses an ultrathin nitrocellulose layer — such as the PATH slides from GenTel BioSciences of Madison, Wisconsin - which maintains binding capacity but reduces background noise.

More specialized surfaces enable orientationspecific presentation of protein probes, such as nickel-NTA (nitrilotriacetic acid) surfaces for use with polyhistidine-tagged proteins. Lumera in Bothell, Washington, uses a protein-protein interaction-based immobilization format for its arrays. "We have a proprietary protein-tag technology that's composed of two coiled coils that bind together with picomolar affinity," says product group manager Ronald Dudek. "One can be engineered into a protein or antibody library, the other is part of the surface chemistry." The pairing of streptavidin-coated slides with biotinylated proteins is also widely used.

\section{Seeing the light}

One aspect of arrays that has changed relatively little is the dominance of fluorescence as a method of detection. "People generally use the standard $\mathrm{Cy} 3$ and $\mathrm{Cy} 5$ fluorophores, which are cheap, reproducible, and scanners are already configured to read them," says Petricoin. His group has done initial studies with semiconductor quantum dots, which offer the benefits of robust long-term fluorescence and enhanced potential for multiplexing. But for many investigators, generically reactive fluorescent dyes are sufficient. "In some ways, labelling is easier than with DNA," says Schena, "because proteins are naturally highly reactive with fluorescent reagents."

Some manufacturers improve the quality of fluorescent-array experiments with specialized instrumentation. The ZeptoREADER from Zeptosens in Witterswil, Switzerland, uses 'planar waveguide' technology, in which an evanescent electromagnetic field is generated by directing light into a specially designed array substrate through a diffraction grating. ${ }^{\alpha}$ The key benefit is that you excite only fluorophores that are bound on the surface," says managing director Markus Ehrat. "In many conventional assays, you don't need to separate bound from unbound fluorophores." Alternatively, in PamGene's flow-through system, samples are repeatedly cycled through porous arrays while being imaged by a sensitive charge-coupled device camera, making it possible to take realtime kinetic measurements.

For experiments involving particularly small samples or scarce protein targets, it might be necessary to amplify the signal. One way to do this is rolling-circle amplification (RCA), first adapted for use with protein arrays by David Ward at Yale University. In RCA, circular DNA molecules are hybridized to capture probes that are conjugated to a detection antibody. These are then repeatedly replicated by DNA

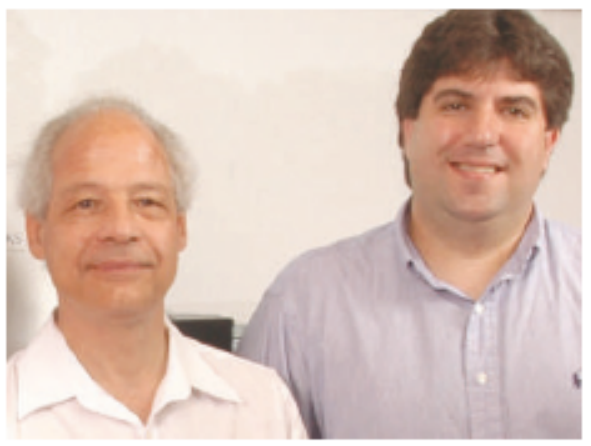

Lance Liotta (left) and Emanuel Petricoin use reverse-phase arrays instead of sandwich arrays in their clinicalresearch.

polymerase and detected with complementary fluorescent probes. Another alternative involves enhanced chemiluminescent detection with tyramide signal amplification, a system available from PerkinElmer of Wellesley, Massachusetts.

As effective as these techniques have proven, they all require a degree of tagging or modification, which can be time-consuming or even impractical for certain samples, such as clinically derived preparations. So many researchers are keeping their eyes on the evolution of 'label-free' technologies that could greatly simplify future studies (see 'Losing the label').

As with genomic arrays, the earliest application - and still the main use - of protein arrays has been the detection or quantification of targets from a research sample or clinical preparation. This typically involves a 'capture array'

\title{
AN APT SOLUTION?
}

Larry Gold has a solution for the problems faced by users of antibody arrays - stop using antibodies. Gold, founder and chief executive of SomaLogic in Boulder, Colorado, is outspoken in his belief that aptamers - smallnucleicacid molecules with specialized functional properties - should replace antibodiesin capture arrays. Years ago, Gold and his team tried to calculate how much multiplexing could be done with an antibody array before background noise became a significant issue. "We guessed thats and wiched antibody arrays were going to start running into serious noise problems when there were $\mathbf{2 0}$ analytes per array," Gold says. He thinks that aptamers could providea level of specificity that surpasses what most antibodies achieve.

More than 15 years ago, Gold was one of the inventors of a now widely used proced ure called SELEX - systematic evolution of ligands by exponentialenrichment. In this system, multiple rounds of selection and amplific ation can beused to select for DNA or RNA molecules with high specificity for a target of choice.

Gold and his colleagues have since enh anced the procedure, and Somalogic uses a high-throughput version of SELEX to generate 'photoaptamers', which can be covalen thy crosslinked to bound targets following irradiation. As a result, aptamers with high affinity and specific crosslinking can beused to measure proteins in complex samples without needing the extra specificity provided by secondary antibodies.

Another advantage of the aptamer platform is simplicity of detection - once protein targets are bound, they can be labelled with ageneric protein-binding fluorescent dye, elimin ating the need for analyte-specific sandwich detection reagents.
Early platform tests have been promising - SomaLogic is achieving successrates of about $80 \%$, Gold says, and he believes a product launch is imminent. "With the right partner, we could launch a product for the research market within some months," he says, "but we think diagnosticsis key, and we're even more interested in transforming evidence-based healthcare."

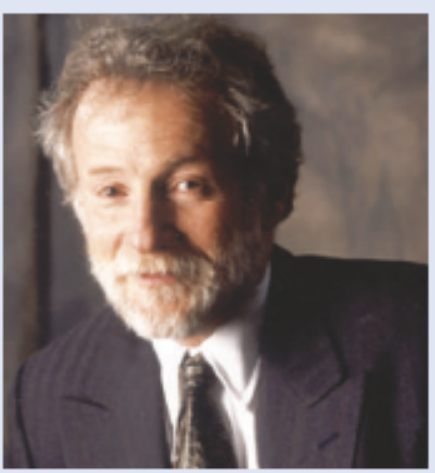

Larry Goldbelieves his aptamer arrays will succeed in applications where antibody arrays fall short.
Some antibody experts are impressed by aptamers, but doubt whether they will unseat the current king "What Larry Gold and others have shown is that when it works, it works very, very well," says Mathias Uhlén of the Royal Institute of Technology in Stockholm, Sweden, "although I would be surprised if aptamers are the dominant scaffold in the future, due to limitations in the chemicalspace."

But SomaLogic is banking on the use of novel nucleotides to increase this chemical space, and believes that a full proteome complement of aptamers is unnecessary - so that high specificity against a few thous and well-chosen targets could be more than sufficient. "We think that, for diagnostics, there's so much redundancy in biology thatyou'll be able to do useful biomarker discovery with an incomplete proteome that's still quite large," says Gold. M.E. 